\title{
Exp-Uniform Distribution: Properties and Characterizations
}

\author{
Z. Javanshiri ${ }^{\dagger}$, A. Habibi Rad ${ }^{\dagger, *}$ and G. G. Hamedani ${ }^{\ddagger}$ \\ $\dagger$ Ferdowsi University of Mashhad \\ ¥ Marquette University
}

\begin{abstract}
In this paper, we study properties of exp-uniform distribution and its applications. We provide closed forms for the density function and moments of order statistics and we also discuss estimation of the parameters via the maximum likelihood method. We will present certain characterizations of exp-uniform distribution. The applications of this distribution are illustrated by fitting it to three real data sets and comparing the results with other lifetime distributions. We hope that this distribution will attract wider applications in lifetime models.
\end{abstract}

Keywords. Characterizations; maximum likelihood estimator; order statistics.

MSC 2010: 62E10, 62F10, 62G30.

\section{Introduction}

Barreto-Souza and Simas (2013) defined a class of distributions given by

$$
F(x)= \begin{cases}\frac{1-e^{-\lambda G(x)}}{1-e^{-\lambda}} & \lambda \neq 0 \\ G(x) & \lambda=0\end{cases}
$$

where $G(x)$ is a cumulative distribution function (cdf) and $\lambda \in \mathbb{R}$ is a constant. The cdf $F$ is called exp- $G$ distribution. Barreto-Souza and

\footnotetext{
* Corresponding author
} 
Simas (2013) obtained several mathematical properties of this class of distributions such as Kullback-Leibler divergence, Shannon entropy, moments, order statistics, estimation of parameters and inference for large sample and discussed the two special cases: exp-Weibull and exp-beta distributions. In this article, we assume $G(x)$ is the cdf of the uniform distribution with parameters $a$ and $b$. So, the random variable $X$ is said to have exp-uniform (EU) distribution with cdf, probability density function (pdf) and hazard functions respectively as

$$
\begin{gathered}
F(x)=\frac{1-e^{-\frac{\lambda(x-a)}{b-a}}}{1-e^{-\lambda}}, \quad a \leqslant x \leqslant b, \\
f(x)=\frac{\lambda e^{-\frac{\lambda(x-a)}{b-a}}}{(b-a)\left(1-e^{-\lambda}\right)}, \quad a<x<b,
\end{gathered}
$$

and

$$
h(x)=\frac{\lambda}{(b-a)\left\{1-e^{-\frac{\lambda(b-x)}{b-a}}\right\}}, \quad a<x<b,
$$

for $\lambda \neq 0, a, b \in \mathbb{R}$. For $a=0, b=2 \pi$ and $2 \pi \lambda$ (in place of $\lambda$ ), (2) is called "Wrapped Exponential" and for $a=-\pi, b=\pi$ and $2 \pi \lambda$, it is called "Modified Wrapped Exponential" in Phani et al. (2013).

We provide four possible motivations for discussing EU distribution. The first motivation is that EU distribution is tractable and there are closed forms of hazard function, characteristic function (cf), moments, density function and moments of order statistics unlike exp-Wiebull and exp-beta distributions. Exact MLEs of $a$ and $b$ are also obtained and shown to be consistent. We hope that our findings will attract applicability in reliability.

The second motivation is based on the relationship between a pdf and its hazard function. In life data analysis increasing hazard rate occurs commonly in practice. Such situations are commonly modelled using the Weibull, gamma and gamma exponential (GE) distributions. They are flexible distributions but they do not allow for an increasing hazard function when their pdfs are monotonically decreasing. They are not suitable for data sets which have increasing empirical hazard functions and decreasing histograms but EU distribution can be fitted to data sets of this kind. Also the beta exponential (BE) distribution that was introduced by Nadarjah and Kotz (2006) such as the weibull, gamma and GE distributions are flexible, but they only 
allow for decreasing or constant hazard function when its respective pdf is monotonically decreasing.

Recently, Nadarajah and Haghighi (2011) introduced an extension of the exponential distribution (exp-NH) as an alternative to the gamma, Weibull and GE distributions. The exp-NH distribution allows for an increasing hazard function when its pdf is monotonically decreasing. But there are no closed forms for MLEs. Also, the moments and Rény entropy are calculated approximately.

Our third motivation is that EU distribution can be interpreted as a truncated exponential distribution. Suppose $Y$ is an exponential random variable with parameter $\lambda$. Let $Z=(b-a) Y+a$, then the distribution in Equation (3) is the same as that of $Z$ truncated at $b$. This fact does not mean that EU distribution does not deserve a separate treatment. Truncated distributions are of special interest by themselves. There are many papers about truncated forms of known distributions. Also, the exp-NH distribution can be interpreted as a truncated Weibull distribution.

The final motivation is the characterizations of EU distribution. An investigator will be vitally interested to know if their model fits the requirements of the EU distribution. To this end, one will depend on the characterizations of this distribution which provide conditions under which the underlying distribution is indeed an EU distribution. We present several characterizations of EU distribution with the hope that they can be used by the applied researchers to determine their distribution model.

The rest of the paper is organized as follows. Section 2 is related to the shape of the pdf and the hazard function. The density and moments of order statistics are calculated in Section 3. In Section 4 MLEs of the parameters are derived. We discuss the properties of MLEs in Section 5. Section 6 will deal with certain characterizations of EU distribution. Applications to real data sets are discussed in Section 7 and some conclusions are drawn in Section 8.

\section{Shape}

Consider the shape of (3) and (4). From (3), $f(a)=\frac{\lambda}{(b-a)\left(1-e^{-\lambda}\right)}, f(b)=$ $\frac{\lambda e^{-\lambda}}{(b-a)\left(1-e^{-\lambda}\right)}$. For $\lambda>0, f(x)$ is a decreasing function and for $\lambda<0, f(x)$ is an increasing function.

From $(4), h(a)=\frac{\lambda}{(b-a)\left(1-e^{-\lambda}\right)}$ and $h(x) \longrightarrow+\infty$ as $x \longrightarrow b$. It is also clear 
that $h(x)$ is an increasing function. Plots of the density (3) and hazard function (4) for $a=5, b=1000$ and some values of $\lambda$ are given in Figure 1.
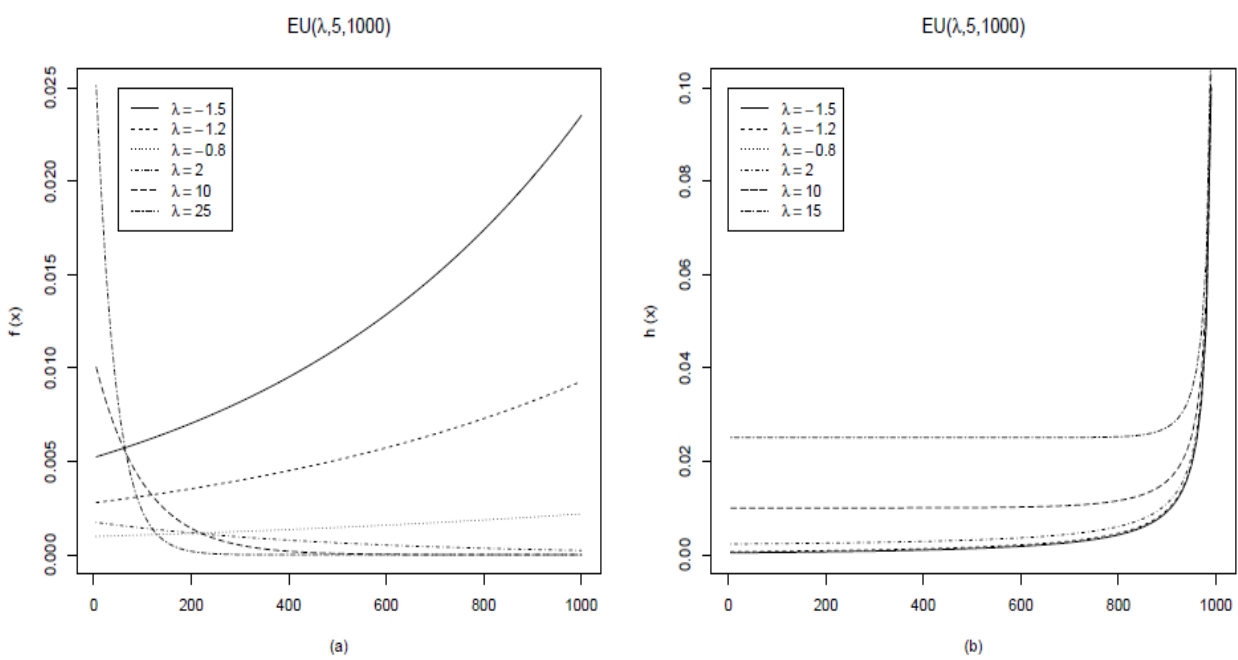

Figure 1. Plots of (a) the density function and (b) the hazard function for $a=5, b=1000$ and some values of $\lambda$.

If $V$ is a random variable with an exponential distribution with parameter $\lambda$, then $X=a-\frac{(b-a)}{\lambda} \ln \left\{e^{-\lambda}+e^{-\lambda V}\left(1-e^{-\lambda}\right)\right\}$ follows EU distribution with parameters $\lambda, a$ and $b$.

\section{Order Statistics}

Here we obtain $f_{i: n}(x)$, the density of the $i$ th order statistic $X_{i: n}$, in a random sample of size $n$ from EU distribution. Let $F(x)$ be an absolutely continuous distribution function with respect to the Lebesgue measure and with pdf $f(x)$. It is well known that

$$
f_{i: n}(x)=\frac{n !}{(i-1) !(n-i) !} f(x) F(x)^{i-1}\{1-F(x)\}^{n-i},
$$

for $i=1, \ldots, n$. Using equations (2) and (3) we have

$$
f_{i: n}(x)=\frac{n ! \lambda e^{-\frac{n \lambda(x-a)}{b-a}}\left\{e^{\frac{\lambda(x-a)}{b-a}}-1\right\}^{i-1}\left\{1-e^{\frac{-\lambda(b-x)}{b-a}}\right\}^{n-i}}{(i-1) !(n-i) !(b-a)\left(1-e^{-\lambda}\right)^{n}} .
$$


Barreto-Souza and Simas (2013) showed that the mean of $X_{i: n}$ is given by

$$
\begin{aligned}
E\left(X_{i: n}\right)= & \frac{\left(1-e^{-\lambda}\right)^{-n}}{B(i, n-i+1)} \\
& \times \sum_{j=0}^{i-1} \sum_{k=0}^{n-i} \frac{(-1)^{n+j-k-1}}{j+k+1}\left(\begin{array}{c}
i-1 \\
j
\end{array}\right)\left(\begin{array}{c}
n-i \\
k
\end{array}\right) e^{-\lambda(n-k-i)} \\
& \times\left\{1-e^{-\lambda(j+k+1)}\right\} E\left(Z_{j, k}\right),
\end{aligned}
$$

where $Z_{j, k}$ has $\operatorname{EU}(\lambda(j+k+1), a, b)$ distribution. Now we give an alternative expression to (6) by the following theorem.

Remark 1. It is easy to see that if $X \sim E U(\lambda, 0,1)$ then $Y=(b-a) X+a \sim$ $E U(\lambda, a, b)$.

Theorem 1. Let $X_{1}, X_{2}, \ldots, X_{n}$ be i.i.d. $E U(\lambda, a, b)$, then

$$
\begin{aligned}
E\left(X_{1: n}\right)= & (b-a)\left\{\frac{1}{\left(1-e^{\lambda}\right)^{n}}+\sum_{k=1}^{n} \frac{1}{k \lambda}+\sum_{j=1}^{n-1} \sum_{k=1}^{n-j} \frac{e^{\lambda}}{j \lambda\left(1-e^{\lambda}\right)^{k}}\right\}+a, \\
E\left(X_{i: n}\right)= & (b-a)\left\{\sum_{k=n-i+1}^{n} \frac{\left(\begin{array}{c}
k-1 \\
k-1-n+i
\end{array}\right) e^{(k-n+i-1) \lambda}(-1)^{k-n+i-1}}{\left(1-e^{\lambda}\right)^{k}}\right. \\
& +\sum_{j=2}^{i-1} \sum_{k=i-j}^{n-j} \frac{(-1)^{i+j} e^{(i-j) \lambda}\left(\begin{array}{c}
k-1 \\
k-i+j
\end{array}\right)}{j \lambda\left(1-e^{\lambda}\right)^{k}}+\frac{(-1)^{i-1}\left(\begin{array}{c}
n-1 \\
n-i
\end{array}\right) e^{(i-1) \lambda}}{\lambda\left(1-e^{\lambda}\right)^{n-1}} \\
& \left.+\sum_{k=i}^{n} \frac{1}{k \lambda}\right\}+a,
\end{aligned}
$$

where $i=2, \ldots, n$ and $n=2,3, \ldots$.

Proof. We take $a=0$ and $b=1$. For general $a$ and $b$, the results follow from Remark 1.

(i) : By equation (5) we have

$$
f_{1: n}(x)=\frac{n \lambda e^{-n \lambda x}\left\{1-e^{-\lambda(1-x)}\right\}^{n-1}}{\left(1-e^{-\lambda}\right)^{n}},
$$

so

$$
\int_{0}^{1} e^{-n \lambda x}\left\{1-e^{-\lambda(1-x)}\right\}^{n-1} d x=\frac{\left(1-e^{-\lambda}\right)^{n}}{n \lambda} .
$$


On taking derivative of both sides of equation (7) with respect to $\lambda$, we have

$$
\begin{aligned}
-\frac{\left(1-e^{-\lambda}\right)^{n}}{\lambda} E\left(X_{1: n}\right)+\frac{e^{-\lambda}\left(1-e^{-\lambda}\right)^{n-1}}{\lambda}\left\{1-E\left(X_{1: n-1}\right)\right\} & =\frac{\left(1-e^{-\lambda}\right)^{n-1}}{\lambda} \\
& \times\left(e^{-\lambda}-\frac{1-e^{-\lambda}}{n \lambda}\right),
\end{aligned}
$$

and hence

$$
E\left(X_{1: n}\right)=\frac{1}{1-e^{\lambda}} E\left(X_{1: n-1}\right)+\frac{1}{n \lambda} .
$$

The above equation is a recursive equation, from which we have

$$
E\left(X_{1: n}\right)=\frac{1}{\left(1-e^{\lambda}\right)^{n}}+\sum_{k=1}^{n} \frac{1}{k \lambda}+\sum_{j=1}^{n-1} \sum_{k=1}^{n-j} \frac{e^{\lambda}}{j \lambda\left(1-e^{\lambda}\right)^{k}} .
$$

(ii): From (5) we have

$$
\int_{0}^{1} \frac{n !}{(n-i) !(i-1) !} e^{-\lambda x n}\left(e^{\lambda x}-1\right)^{i-1}\left\{1-e^{\lambda(x-1)}\right\}^{n-i} d x=\frac{\left(1-e^{-\lambda}\right)^{n}}{\lambda} .
$$

Differentiating (8) with respect to $\lambda$, we have

$$
E\left(X_{i: n}\right)=\frac{-e^{\lambda}}{1-e^{\lambda}} E\left(X_{i-1: n-1}\right)+\frac{1}{1-e^{\lambda}} E\left(X_{i: n-1}\right)+\frac{1}{n \lambda} .
$$

If $i=2$ we obtain

$$
E\left(X_{2: n}\right)=\frac{-e^{\lambda}}{1-e^{\lambda}} E\left(X_{1: n-1}\right)+\frac{1}{1-e^{\lambda}} E\left(X_{2: n-1}\right)+\frac{1}{n \lambda} .
$$

Substituting $E\left(X_{1: n-1}\right)$ from $(i)$, we arrive at

$$
E\left(X_{2: n}\right)=\frac{-e^{\lambda}}{\left(1-e^{\lambda}\right)^{n}}-\sum_{k=1}^{n-1} \frac{e^{\lambda}}{k \lambda\left(1-e^{\lambda}\right)^{n-k}}+\frac{1}{1-e^{\lambda}} E\left(X_{2: n-1}\right)+\frac{1}{n \lambda} .
$$

The above equation is a recursive equation, from which we derive

$$
E\left(X_{2: n}\right)=\frac{-(n-1) e^{\lambda}}{\left(1-e^{\lambda}\right)^{n}}-\frac{(n-1) e^{\lambda}}{\lambda\left(1-e^{\lambda}\right)^{n-1}}+\frac{1}{\left(1-e^{\lambda}\right)^{n-1}}+\sum_{k=2}^{n} \frac{1}{k \lambda} .
$$

If in (9) $i=3$, we have

$$
E\left(X_{3: n}\right)=\frac{-e^{\lambda}}{1-e^{\lambda}} E\left(X_{2: n-1}\right)+\frac{1}{1-e^{\lambda}} E\left(X_{3: n-1}\right)+\frac{1}{n \lambda} .
$$


By substituting $E\left(X_{2: n-1}\right)$ from (10), we deduce

$$
\begin{aligned}
E\left(X_{3: n}\right)= & \frac{(n-2) e^{2 \lambda}}{\left(1-e^{\lambda}\right)^{n}}+\frac{(n-2) e^{2 \lambda}}{\lambda\left(1-e^{\lambda}\right)^{n-1}}-\frac{e^{\lambda}}{\left(1-e^{\lambda}\right)^{n-1}}-\frac{e^{\lambda}}{1-e^{\lambda}} \sum_{k=2}^{n-1} \frac{1}{k \lambda} \\
& +\frac{1}{1-e^{\lambda}} E\left(X_{3: n-1}\right)+\frac{1}{n \lambda} .
\end{aligned}
$$

The above equation is a recursive equation, from which we derive

$$
\begin{aligned}
E\left(X_{3: n}\right)= & \frac{(n-2)(n-1) e^{2 \lambda}}{2\left(1-e^{\lambda}\right)^{n}}+\frac{(n-2)(n-1) e^{2 \lambda}}{2 \lambda\left(1-e^{\lambda}\right)^{n-1}}-\frac{(n-2) e^{\lambda}}{\left(1-e^{\lambda}\right)^{n-1}} \\
& +\frac{1}{\left(1-e^{\lambda}\right)^{n-2}}-\sum_{k=1}^{n-2} \frac{e^{\lambda}}{2 \lambda\left(1-e^{\lambda}\right)^{k}}+\sum_{k=3}^{n} \frac{1}{k \lambda} .
\end{aligned}
$$

Similarly, we obtain

$$
\begin{aligned}
E\left(X_{4: n}\right)= & \frac{-(n-3)(n-2)(n-1) e^{3 \lambda}}{6\left(1-e^{\lambda}\right)^{n}}-\frac{(n-3)(n-2)(n-1) e^{3 \lambda}}{6 \lambda\left(1-e^{\lambda}\right)^{n-1}} \\
& +\sum_{k=2}^{n-2} \frac{(k-1) e^{2 \lambda}}{2 \lambda\left(1-e^{\lambda}\right)^{k}}-\sum_{k=1}^{n-3} \frac{e^{\lambda}}{3 \lambda\left(1-e^{\lambda}\right)^{k}}+\frac{(n-3)(n-2) e^{2 \lambda}}{2\left(1-e^{\lambda}\right)^{n-1}} \\
& -\frac{(n-3) e^{\lambda}}{\left(1-e^{\lambda}\right)^{n-2}}+\frac{1}{\left(1-e^{\lambda}\right)^{n-3}}+\sum_{k=4}^{n} \frac{1}{k \lambda}
\end{aligned}
$$

and thus

$$
\begin{aligned}
E\left(X_{i: n}\right)= & \sum_{k=n-i+1}^{n} \frac{\left(\begin{array}{c}
k-1 \\
k-1-n+i
\end{array}\right) e^{(k-n+i-1) \lambda}(-1)^{k-n+i-1}}{\left(1-e^{\lambda}\right)^{k}} \\
& +\sum_{j=2}^{i-1} \sum_{k=i-j}^{n-j} \frac{(-1)^{i+j} e^{(i-j) \lambda}\left(\begin{array}{c}
k-1 \\
k-i+j
\end{array}\right)}{j \lambda\left(1-e^{\lambda}\right)^{k}} \\
& +\frac{(-1)^{i-1}\left(\begin{array}{c}
n-1 \\
n-i
\end{array}\right) e^{(i-1) \lambda}}{\lambda\left(1-e^{\lambda}\right)^{n-1}}+\sum_{k=i}^{n} \frac{1}{k \lambda}
\end{aligned}
$$




\section{Parameter Estimation}

Suppose $X_{1}, \ldots, X_{n}$ constitute a random sample from an EU distribution with density (3). The likelihood function is given by

$$
L(\lambda, a, b)=\frac{\lambda^{n} e^{\frac{-\lambda}{b-a} \sum_{i=1}^{n}\left(x_{i}-a\right)}}{(b-a)^{n}\left(1-e^{-\lambda}\right)^{n}}, \quad a<x_{1: n}<x_{2: n}<\cdots<x_{n: n}<b,
$$

so, the log-likelihood function is

$$
\begin{aligned}
\ell(\lambda, a, b)= & \left\{n \ln \left(\frac{\lambda}{1-e^{-\lambda}}\right)-n \ln (b-a)-\frac{\lambda n(\bar{x}-a)}{b-a}\right\} \\
& \times I_{\left(-\infty, x_{1: n}\right)}(a) I_{\left(x_{n: n},+\infty\right)}(b) .
\end{aligned}
$$

Taking partial derivative of the log-likelihood in (11) with respect to $a, b$ and $\lambda$, respectively,

$$
\begin{aligned}
\frac{\partial \ell}{\partial a} & =\left\{\frac{n(b-a)-\lambda n(\bar{x}-b)}{(b-a)^{2}}\right\} I_{\left(-\infty, x_{1: n}\right)}(a) \\
\frac{\partial \ell}{\partial b} & =\left\{\frac{-n}{b-a}+\frac{n \lambda(\bar{x}-a)}{(b-a)^{2}}\right\} I_{\left(x_{n: n},+\infty\right)}(b), \\
\frac{\partial \ell}{\partial \lambda} & =\frac{n}{\lambda}-\frac{n e^{-\lambda}}{1-e^{-\lambda}}-\frac{n(\bar{x}-a)}{b-a}=0 .
\end{aligned}
$$

From (12) and (14), $\ell$ is increasing in $a$ when $a<x_{1: n}$ and is 0 otherwise. So the MLE of $a$ is $X_{1: n}$. Also (14) and (13) yield $\ell$ is decreasing in $b$ when $b>x_{n: n}$ and is 0 otherwise. Hence the MLE of $b$ is $X_{n: n}$. Substituting $a=X_{1: n}$ and $b=X_{n: n}$ into equation (14) follows

$$
h(\lambda)=\frac{\bar{x}-x_{1: n}}{x_{n: n}-x_{1: n}}
$$

where $h(\lambda)=\frac{1}{\lambda}-\frac{1}{e^{\lambda}-1}$. Note that $h(\lambda)$ is a decreasing continuous function, $\lim _{\lambda \rightarrow-\infty} h(\lambda)=1$ and $\lim _{\lambda \rightarrow+\infty} h(\lambda)=0$. Hence equation (15) has a unique solution. Since $\frac{\partial^{2} \ell}{\partial \lambda^{2}}$ is negative, this unique solution is the MLE of $\lambda$. The solution of equation (15) can be evaluated numerically by Newton-Raphson method. 


\section{Properties of MLEs}

In this section we will consider the asymptotic properties of the MLEs. First, we examine the consistency of estimators. In view of

$$
\begin{gathered}
\lim _{n \rightarrow+\infty} P\left\{\left|X_{1: n}-a\right|>\epsilon\right\}=\lim _{n \rightarrow+\infty}\left\{\frac{e^{\frac{-\lambda \epsilon}{b-a}}-e^{-\lambda}}{1-e^{-\lambda}}\right\}^{n}=0, \\
\lim _{n \rightarrow+\infty} P\left\{\left|X_{n: n}-b\right|>\epsilon\right\}=\lim _{n \rightarrow+\infty}\left\{\frac{1-e^{-\lambda\left(1-\frac{\epsilon}{b-a}\right)}}{1-e^{-\lambda}}\right\}^{n}=0,
\end{gathered}
$$

we conclude that $X_{1: n}$ and $X_{n: n}$ are consistent for $a$ and $b$, respectively.

If the suitable regularity conditions hold and the likelihood equation has a unique root, the solution of the likelihood equation is the MLE which is consistent (Lehmann and Casella, 1998). When $a$ and $b$ are known, the regularity conditions hold and from Section 4, the MLE of $\lambda$ is unique and hence consistent. This is also true when $a$ and $b$ are unknown, since $X_{1: n}$ and $X_{n: n}$ are consistent.

Next, we derive asymptotic distributions of the MLEs. From

$$
\begin{gathered}
P\left\{n\left(X_{1: n}-a\right) \leqslant t\right\}=1-\left\{1-\frac{1-e^{\frac{-\lambda t}{n(b-a)}}}{1-e^{-\lambda}}\right\} \stackrel{n}{\rightarrow} 1-e^{\frac{-\lambda t}{(b-a)\left(1-e^{-\lambda}\right)}} \\
P\left\{n\left(b-X_{n: n}\right) \leqslant t\right\}=1-\left[1+\frac{e^{-\lambda}\left\{1-e^{\frac{-\lambda t}{n(b-a)}}\right\}}{1-e^{-\lambda}}\right]^{n} \stackrel{d}{\rightarrow} 1-e^{\frac{-e^{-\lambda} \lambda t}{(b-a)\left(1-e^{-\lambda}\right)}}
\end{gathered}
$$

as $n \rightarrow+\infty$, we conclude that $n\left(X_{1: n}-a\right) \stackrel{d}{\rightarrow} \exp \left\{\frac{\lambda}{(b-a)\left(1-e^{-\lambda}\right)}\right\}$ and $n(b-$ $\left.X_{n: n}\right) \stackrel{d}{\rightarrow} \exp \left\{\frac{\lambda e^{-\lambda}}{(b-a)\left(1-e^{-\lambda}\right)}\right\}$. So $X_{1: n}$ and $X_{n: n}$ are asymptotically biased. If $a$ and $b$ are known, the regularity conditions hold and $\sqrt{n}(\hat{\lambda}-\lambda) \stackrel{d}{\rightarrow}$ $N\left(0, \frac{\lambda^{2}\left(e^{\lambda}-1\right)^{2}}{\left(e^{\lambda}-1\right)^{2}-\lambda^{2} e^{\lambda}}\right)$. This is also true when $a$ and $b$ are unknown, since $X_{1: n}$ and $X_{n: n}$ are consistent.

\section{Characterizations}

The problem of characterizing a distribution is an important problem which has recently attracted the attention of many researchers. Thus, various char- 
acterizations have been established in many different directions. An investigator will be vitally interested to know if their model fits the requirements of the EU distribution. To this end, one will depend on the characterizations of this distribution which provide conditions under which the underlying distribution is indeed an EU distribution. In this section, several characterizations of EU distribution are presented. These characterizations are based on: (i) a simple relationship between two truncated moments, (ii) conditional expectation of certain functions of the random variable, (iii) hazard function.

\subsection{Characterization Based on Two Truncated Moments}

In this subsection we present characterizations of cdf given by (2) in terms of a simple relationship between two truncated moments. We like to mention here the works of Glänzel and Hamedani (2001) and Hamedani (1993, 2002, 2006, 2010) as well as references therein, in this direction. Our characterization results presented here will employ an interesting result due to Glänzel (1987) (Theorem 2 below).

Theorem 2. Let $(\Omega, \mathcal{F}, \mathbf{P})$ be a given probability space and let $H=[a, b]$ be an interval for some $a<b(a=-\infty, b=+\infty$ might as well be allowed $)$. Let $X: \Omega \longrightarrow H$ be a continuous random variable with the distribution function $F$ and let $g$ and $h$ be two real functions defined on $H$ such that

$$
E\{g(X) \mid X \geqslant x\}=E\{h(X) \mid X \geqslant x\} \eta(x), \quad x \in H,
$$

is defined with some real function $\eta$. Assume that $g, h \in \mathcal{C}^{1}(H), \eta \in \mathcal{C}^{2}(H)$ and $F$ is twice continuously differentiable and strictly monotone function on the set $H$. Finally, assume that the equation $h \eta=g$ has no real solution in the interior of $H$. Then $F$ is uniquely determined by functions $g, h$ and $\eta$, particularly

$$
F(x)=\int_{a}^{x} \mathcal{C}\left|\frac{\eta^{\prime}(u)}{\eta(u) h(u)-g(u)}\right| \exp \{-s(u)\} d u
$$

where the function $s$ is a solution of the differential equation $s^{\prime}=\frac{\eta^{\prime} h}{\eta h-g}$ and $\mathcal{C}$ is a constant, chosen to make $\int_{H} d F=1$.

Remark 2. (a) In Theorem 2, the interval $H$ need not be closed. (b) The goal is to have the function $\eta$ as simple as possible. For a more detailed 
discussion on the choice of $\eta$, we refer the reader to Glänzel and Hamedani (2001) and Hamedani (1993, 2002, 2006, 2010).

Proposition 1. Let $X: \Omega \longrightarrow(a, b)$ be a continuous random variable and let $h(x)=1$ and $g(x)=\exp \left\{-\lambda \frac{(x-a)}{b-a}\right\}$ for $x \in(a, b)$. The pdf of $X$ is (3) if and only if the function $\eta$ defined in Theorem 2 has the form

$$
\eta(x)=\frac{1}{2}\left\{e^{-\lambda \frac{(x-a)}{b-a}}+e^{-\lambda}\right\}, \quad x \in(a, b) .
$$

Proof. Let $X$ have pdf (3), then

$$
\{1-F(x)\} E\{h(X) \mid X \geqslant x\}=\left\{\frac{e^{-\lambda \frac{(x-a)}{b-a}}-e^{-\lambda}}{1-e^{-\lambda}}\right\}, \quad x \in(a, b),
$$

and

$$
\{1-F(x)\} \mathbf{E}\{g(X) \mid X \geqslant x\}=\frac{1}{2}\left\{\frac{e^{-2 \lambda \frac{(x-a)}{b-a}}-e^{-2 \lambda}}{1-e^{-\lambda}}\right\}, \quad x \in(a, b),
$$

and finally

$$
\eta(x) h(x)-g(x)=\frac{1}{2}\left\{e^{-\lambda}-e^{-\lambda \frac{(x-a)}{b-a}}\right\}<0, \quad x \in(a, b) .
$$

Conversely, if $\eta$ is given as above, then

$$
s^{\prime}(x)=\frac{\eta^{\prime}(x) h(x)}{\eta(x) h(x)-g(x)}=\frac{\lambda e^{-\lambda \frac{(x-a)}{b-a}}}{(b-a)\left\{e^{-\lambda \frac{(x-a)}{b-a}}-e^{-\lambda}\right\}}, \quad x \in(a, b)
$$

and hence

$$
s(x)=-\ln \left\{e^{-\lambda \frac{(x-a)}{b-a}}-e^{-\lambda}\right\}+C_{1}, \quad x \in(a, b),
$$

where $C_{1}$ is a constant. Now, in view of Theorem $2, \mathrm{X}$ has cdf (2) and pdf (3). 
Corollary 1. Let $X: \Omega \longrightarrow(a, b)$ be a continuous random variable and let $h(x)$ be as in Proposition 1. The pdf of $X$ is (3) if and only if there exist functions $g$ and $\eta$ defined in Theorem 2, satisfying the differential equation

$$
\frac{\eta^{\prime}(x)}{\eta(x)-g(x)}=\frac{\lambda e^{-\lambda \frac{x-a}{b-a}}}{(b-a)\left(e^{-\lambda \frac{x-a}{b-a}}-e^{-\lambda}\right)}, \quad x \in(a, b) .
$$

Remark 3. (c) The general solution of the differential equation in Corollary 1 is

$$
\eta(x)=(b-a)^{-1}\left(e^{-\lambda \frac{x-a}{b-a}}-e^{-\lambda}\right)^{-1}\left\{-\int g(x) \lambda e^{-\lambda \frac{x-a}{b-a}} d x+D\right\},
$$

for $x \in(a, b)$, where $D$ is a constant. One set of appropriate functions is given in Proposition 1 with $D=\frac{b-a}{2} e^{-2 \lambda}$. (d) clearly there are other triplets of functions $(h, g, \eta)$ satisfying the conditions of Theorem 2. We presented one such triplet in Proposition 1.

\subsection{Characterization Based on Conditional Expectation of Certain Function of the Random Variable}

In this subsection we employ a single function $\psi$ of $X$ and characterize the distribution of $X$ in terms of the conditional expectation of $\psi(X)$. The following propositions have already appeared in our previous work (as a technical report), so we will just state them here for the sake of completeness.

Proposition 2. Let $X: \Omega \longrightarrow(a, b)$ be a continuous random variable with cdf $F$. Let $\psi(x)$ be a differentiable function on $(a, b)$ with $\lim _{x \longrightarrow b} \psi(x)=1$. Then for $\delta \neq 1$,

$$
E[\psi(X) \mid X>x]=\delta \psi(x), \quad x \in(a, b),
$$

if and only if

$$
\psi(x)=\{1-F(x)\}^{\frac{1}{\delta}-1}, \quad x \in(a, b) .
$$


Proposition 3. Let $X: \Omega \longrightarrow(a, b)$ be a continuous random variable with cdf $F$. Let $\psi_{1}(x)$ be a differentiable function on $(a, b)$ with $\lim _{x \rightarrow a} \psi_{1}(x)=1$. Then for $\delta_{1} \neq 1$,

$$
E\left\{\psi_{1}(X)<x\right\}=\delta_{1} \psi_{1}(x), \quad x \in(a, b),
$$

if and only if

$$
\psi_{1}(x)=\{F(x)\}^{\frac{1}{\delta_{1}}-1}, \quad x \in(a, b) .
$$

Remark 4. (e) For $\psi(x)=\left\{\frac{e^{-\lambda \frac{(x-a)}{b-a}}-e^{-\lambda}}{1-e^{-\lambda}}\right\}^{\frac{1-\delta}{\delta}}, x \in(a, b)$, Proposition 2 will give a cdf $F(x)$ given by $(2)$. ( $(f)$ For $\psi_{1}(x)=\left\{\frac{1-e^{-\lambda} \frac{(x-a)}{b-a}}{1-e^{-\lambda}}\right\}^{\frac{1-\delta_{1}}{\delta_{1}}}$, $x \in(a, b)$, Proposition 3 will give a $\operatorname{cdf} F(x)$ given by $(2)$.

\subsection{Characterization Based on Hazard Function}

For the sake of completeness, we state the following definition.

Definition 1. Let $F$ be an absolutely continuous distribution with the corresponding pdf $f$. The hazard function corresponding to $F$ is denoted by $\eta_{F}$ and is defined by

$$
\eta_{F}(x)=\frac{f(x)}{1-F(x)}, \quad x \in \operatorname{Supp} F,
$$

where $\operatorname{Supp} F$ is the support of $F$.

It is obvious that the hazard function of a twice differentiable distribution function satisfies the first order differential equation

$$
\frac{\eta_{F}^{\prime}(x)}{\eta_{F}(x)}-\eta_{F}(x)=q(x),
$$

where $q(x)$ is an appropriate integrable function. Although this differential equation has an obvious form since

$$
\frac{f^{\prime}(x)}{f(x)}=\frac{\eta_{F}^{\prime}(x)}{\eta_{F}(x)}-\eta_{F}(x),
$$


for many univariate continuous distributions (16) seems to be the only differential equation in terms of the hazard function. The goal of the characterizations based on hazard function is to establish a differential equation in terms of hazard function, which has as simple form as possible and is not of the trivial form (16). For some general families of distributions this may not be possible. Here we present a characterization of a EU distribution based on a nontrivial differential equation in terms of the hazard function.

Proposition 4. Let $X: \Omega \rightarrow(0, \infty)$ be a continuous random variable. The pdf of $X$ is (3) if and only if its hazard function $\eta_{F}(x)$ satisfies the differential equation

$$
\eta_{F}^{\prime}(x)=e^{\frac{\lambda(x-b)}{b-a}} \eta_{F}^{2}(x), \quad a<x<b,
$$

with boundary condition $\eta_{F}(a)=\frac{\lambda}{b-a}\left(1-e^{-\lambda}\right)^{-1}$.

Proof. If $X$ has pdf (3), then clearly (19) holds. Now, if (19) holds, then

$$
\frac{\eta_{F}^{\prime}(x)}{\eta_{F}^{2}(x)}=e^{\frac{\lambda(x-b)}{b-a}}
$$

from which we have

or

$$
\frac{d}{d x}\left\{\frac{1}{\eta_{F}(x)}\right\}=\frac{d}{d x}\left[\frac{b-a}{\lambda}\left\{1-e^{\frac{\lambda(x-b\}}{b-a}}\right\}\right],
$$

$$
\eta_{F}(x)=\frac{b-a}{\lambda}\left\{1-e^{\frac{\lambda(x-b)}{b-a}}\right\}^{-1}=\frac{b-a}{\lambda}\left\{\frac{e^{\frac{-\lambda(x-a)}{b-a}}}{e^{\frac{-\lambda(x-b)}{b-a}}-e^{-\lambda}}\right\} .
$$

Integrating both sides of (20) from $a$ to $x$, we arrive at

$$
\begin{aligned}
-\ln \{1-F(x)\} & =\int_{a}^{x} \frac{\frac{b-a}{\lambda} e^{\frac{-\lambda(u-a)}{b-a}}}{e^{\frac{-\lambda(u-b)}{b-a}}-e^{-\lambda}} d u \\
& =-\ln \left\{e^{\frac{-\lambda(x-a)}{b-a}}-e^{-\lambda}\right\}+\ln \left(1-e^{-\lambda}\right) .
\end{aligned}
$$

From the last equality, we obtain

$$
1-F(x)=\frac{e^{\frac{-\lambda(x-a)}{b-a}}-e^{-\lambda}}{1-e^{-\lambda}}, \quad a \leqslant x \leqslant b .
$$




\section{Applications}

In this section we fit EU model to real data sets. We provide three applications.

Application 1. Proschan (1963) provides the times, in hours of operation, between successive failures of air conditioning equipment in 13 aircraft. The data for plane number 3 are: 90, 10, 60, 186, 61, 49, 14, 24, 56, 20, 79, 84, 44, $59,29,118,25,156,310,76,26,44,23,62,130,208,70,101,208$. Lawless (2003, page 477) showed a Weibull distribution fits to this data. Now, we fit the GE, gamma, exponentiated Weibull (E-Weibull), generalized gamma (G-gmma) and EU distributions to data. The MLEs of the parameters and the maximized log-likelihood $(\hat{\ell})$ for this distributions are derived.

The results of goodness of fit tests based on bootstrap Kolmogorov -Smirnov test and the evaluation of the corrected Akaike information criterion $\left(A I C_{c}\right)$ (Sugiura, 1978; Hurvich and Tsai, 1989) are shown in Table 1.

Table 1. Goodness-of-fit test based on bootstrap Kolmogorov-Smirnov test and $A I C_{c}$ for plane 3 .

\begin{tabular}{lcccc}
\hline \hline \multicolumn{1}{c}{ Model } & Estimated Parameters & $\hat{\ell}$ & P-value & $A I C_{c}$ \\
\hline Weibull $(\alpha, \lambda)$ & $\hat{\alpha}=1.29, \quad \hat{\lambda}=90.65$ & -155.84 & 0.703 & 316.14 \\
$\operatorname{GE}(\lambda, \alpha)$ & $\hat{\lambda}=0.017, \quad \hat{\alpha}=1.8$ & -155.22 & 0.782 & 314.90 \\
$\operatorname{gamma}(\alpha, \lambda)$ & $\hat{\alpha}=1.67, \hat{\lambda}=0.02$ & -155.35 & 0.781 & 315.15 \\
$\operatorname{E-Weibull}(k, \lambda, \alpha)$ & $\hat{k}=0.555, \hat{\lambda}=12.841, \hat{\alpha}=7.16$ & -154.75 & 0.745 & 316.45 \\
$\operatorname{G-gamma}(a, b, k)$ & $\hat{a}=0.274, \hat{b}=0.0013, \hat{k}=19.312$ & -154.72 & 0.750 & 316.40 \\
$\operatorname{EU}(\lambda, a, b)$ & $\hat{\lambda}=3.7, \hat{a}=10, \hat{b}=310$ & -153.04 & 0.943 & 313.03 \\
\hline
\end{tabular}

From the Table 1, we see that EU distribution is a better fit, judging on the basis of $A I C_{c}$ s. The probability plots given in Figure 2, also show that EU distribution gives a better fit than the other distributions.

We also consider the data for plane number 7: 97, 51, 11, 4, 141, 18, 142, $68,77,80,1,16,106,206,82,54,31,216,46,111,39,63,18,191,18,163$, 24. The similar results are shown in Table 2 . 

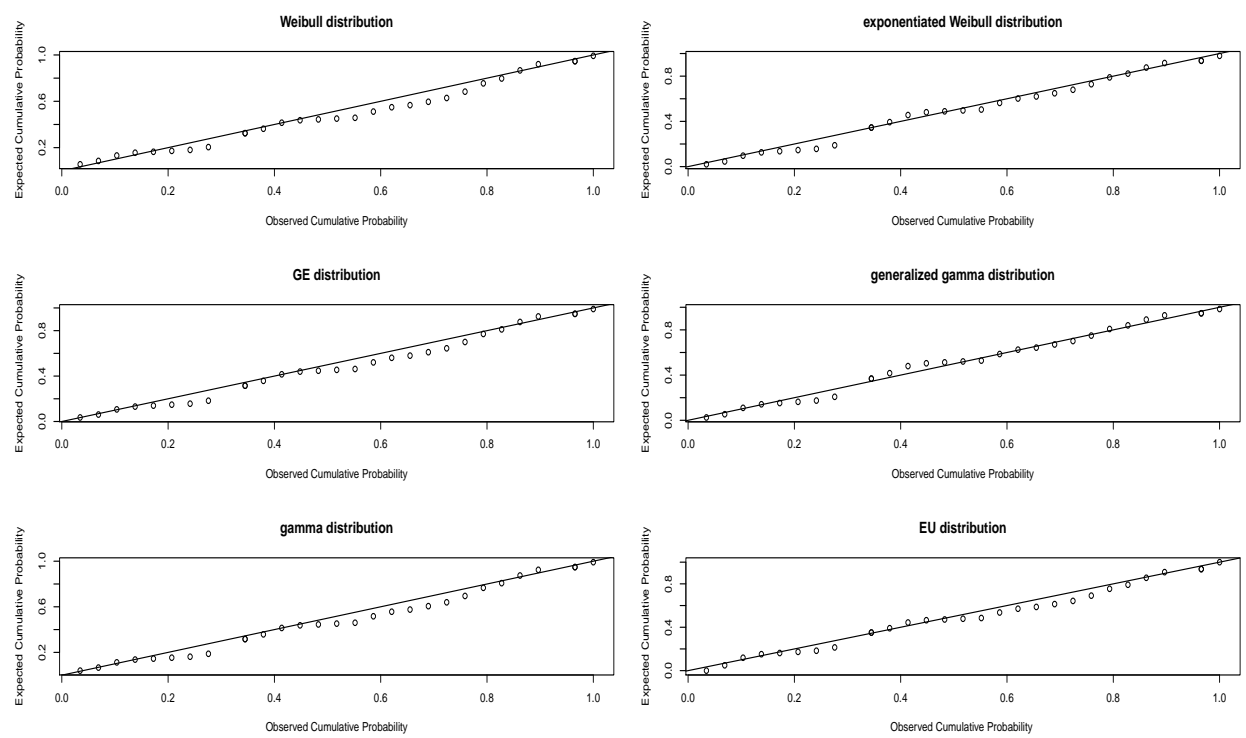

Figure 2. Probability plots for the fit of the Weibull, gamma, GE, E-Weibull, Ggamma and EU distributions to the data for plane 3 .

Table 2. Goodness-of-fit test based on bootstrap Kolmogorov-Smirnov test and $A I C_{c}$ for plane 7 .

\begin{tabular}{lcccc}
\hline \hline \multicolumn{1}{c}{ Model } & Estimated Parameters & $\hat{\ell}$ & P-value & $A I C_{c}$ \\
\hline Weibull $(\alpha, \lambda)$ & $\hat{\alpha}=1.12, \quad \hat{\lambda}=80.185$ & -143.96 & 0.843 & 292.41 \\
$\operatorname{GE}(\lambda, \alpha)$ & $\hat{\lambda}=0.013, \quad \hat{\alpha}=1.12$ & -144.12 & 0.975 & 292.73 \\
$\operatorname{gamma}(\alpha, \lambda)$ & $\hat{\alpha}=1.13, \hat{\lambda}=0.014$ & -144.09 & 0.957 & 292.68 \\
$\operatorname{E-Weibull}(k, \lambda, \alpha)$ & $\hat{k}=3.18, \hat{\lambda}=166.62, \hat{\alpha}=0.237$ & -143.29 & 0.735 & 293.63 \\
$\operatorname{G-gamma}(a, b, k)$ & $\hat{a}=3.28, \hat{b}=186.87, \hat{k}=0.231$ & -143.33 & 0.853 & 293.71 \\
$\operatorname{EU}(\lambda, a, b)$ & $\hat{\lambda}=1.87, \hat{a}=1, \hat{b}=216$ & -141.39 & 0.809 & 289.82 \\
\hline
\end{tabular}

From the Table 2, we see that in all cases the P-values are high, we cannot reject the null hypothesis that data are coming from the Weibull, GE, gamma, E-Weibull, G-gamma and EU distributions. We compare models based on $A I C_{c}$. We prefer EU distribution, since its $A I C_{C}$ is lowest. The probability plots are given in Figure 3. 

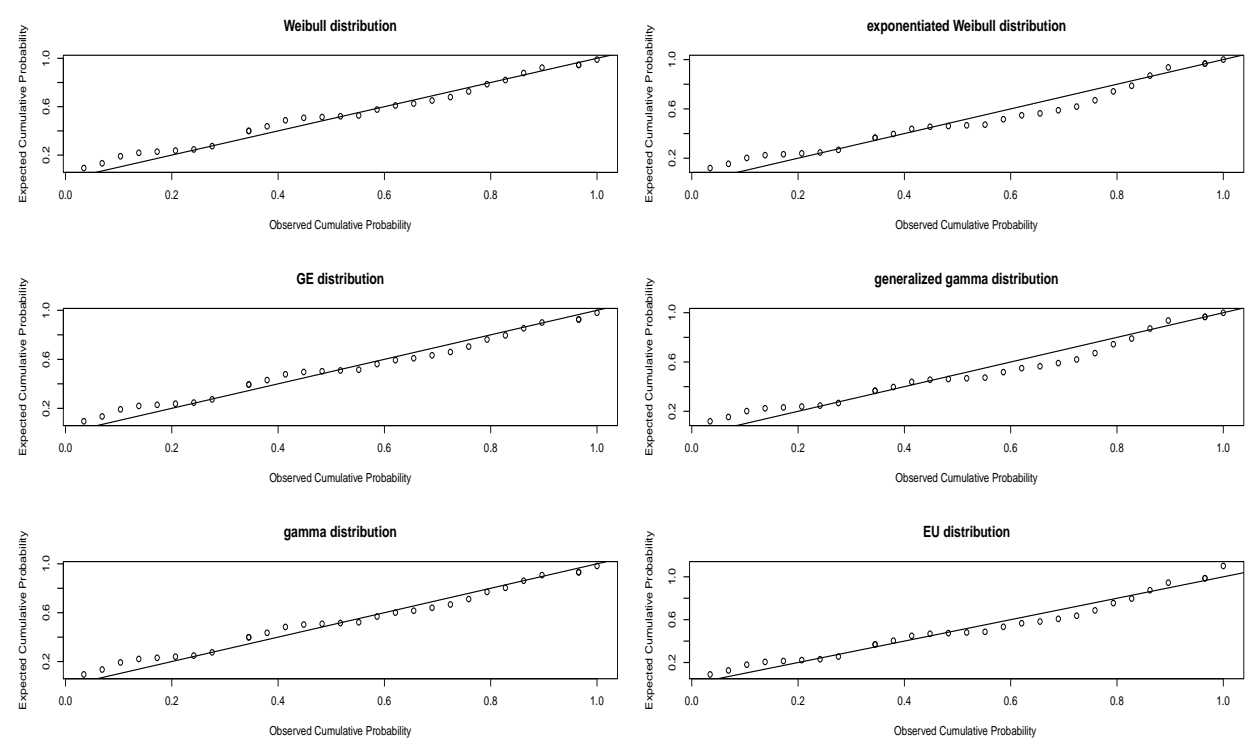

Figure 3. Probability plots for the fit of the Weibull, gamma, GE, E-Weibull, G-gamma and EU distributions to the data for plan 7 .

Application 2. Crowder (2000) provides the lifetimes of steel specimens tested at 14 different stress levels. The data for level 32 are: 60, 51, 83, 140, 109, 106, 119, 76, 68, 67, 111, 57, 69, 75, 122, 128, 95, 87, 82, 132. We consider four possible models for the data set: the Weibull, GE, gamma, E-Weibull, G-gamma and EU distributions. The results of goodness of fit tests based on bootstrap Kolmogorov-Smirnov test and $A I C_{c}$ are shown in Table 3 .

Table 3. Goodness-of-fit test based on bootstrap Kolmogorov-Smirnov test and $A I C_{c}$.

\begin{tabular}{lcccc}
\hline \hline \multicolumn{1}{c}{ Model } & Estimated Parameters & $\hat{\ell}$ & P-value & $A I C_{c}$ \\
\hline Weibull $(\alpha, \lambda)$ & $\hat{\alpha}=3.84, \hat{\lambda}=101.79$ & -96.27 & 0.447 & 197.239 \\
$\operatorname{GE}(\lambda, \alpha)$ & $\hat{\lambda}=0.044, \hat{\alpha}=31.02$ & -93.65 & 0.378 & 192.003 \\
$\operatorname{gamma}(\alpha, \lambda)$ & $\hat{\alpha}=11.37, \hat{\lambda}=0.124$ & -93.52 & 0.575 & 191.740 \\
$\operatorname{E-Weibull}(k, \lambda, \alpha)$ & $\hat{k}=0.507, \hat{\lambda}=1.63, \hat{\alpha}=122.65$ & -93.92 & 0.277 & 195.34 \\
$\operatorname{G-gamma}(a, b, k)$ & $\hat{a}=0.672, \hat{b}=0.719, \hat{k}=25.74$ & -93.51 & 0.417 & 194.52 \\
$\operatorname{EU}(\lambda, a, b)$ & $\hat{\lambda}=0.49, \hat{a}=51, \hat{b}=140$ & -89.57 & 0.941 & 186.64 \\
\hline
\end{tabular}


From the Table 3, we see that in all cases, we cannot reject the null hypothesis that data are coming from the Weibull, GE, gamma, E-Weibull, G-gamma and EU distributions. We compare these models by Akaike's method. The $A I C_{c}$ for EU model is lowest so we prefer EU model. The plots of the estimated pdfs of the Weibull, GE, gamma, E-Weibull, G-gamma and EU distributions fitted to data set and corresponding probability plots are given in Figure 4 and Figure 5, respectively. These Figures show that EU distribution gives a better fit than the other models.

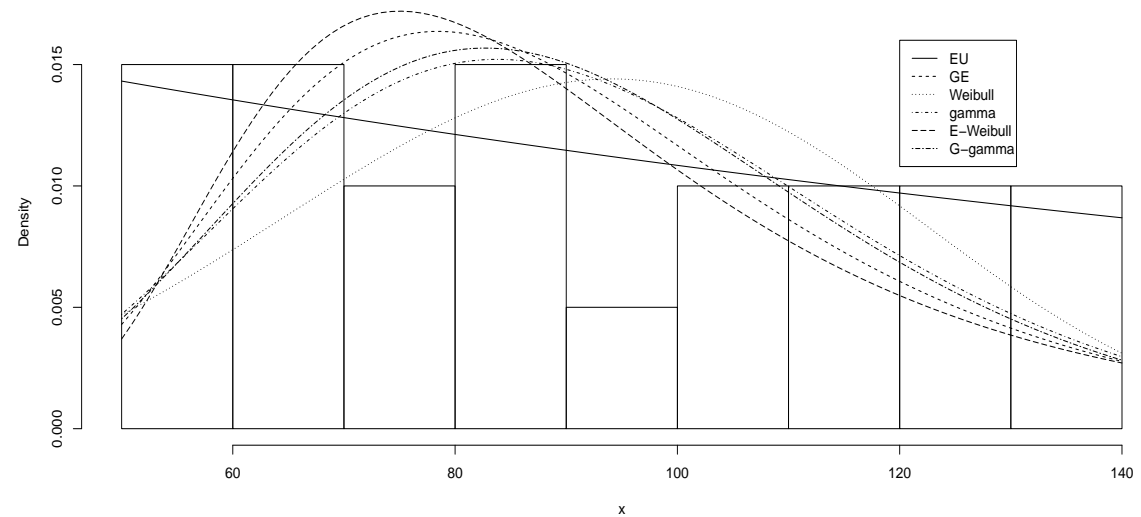

Figure 4. Estimated pdfs of EU, GE, Weibull, gamma, E-Weibull and G-gamma distributions for the data set.
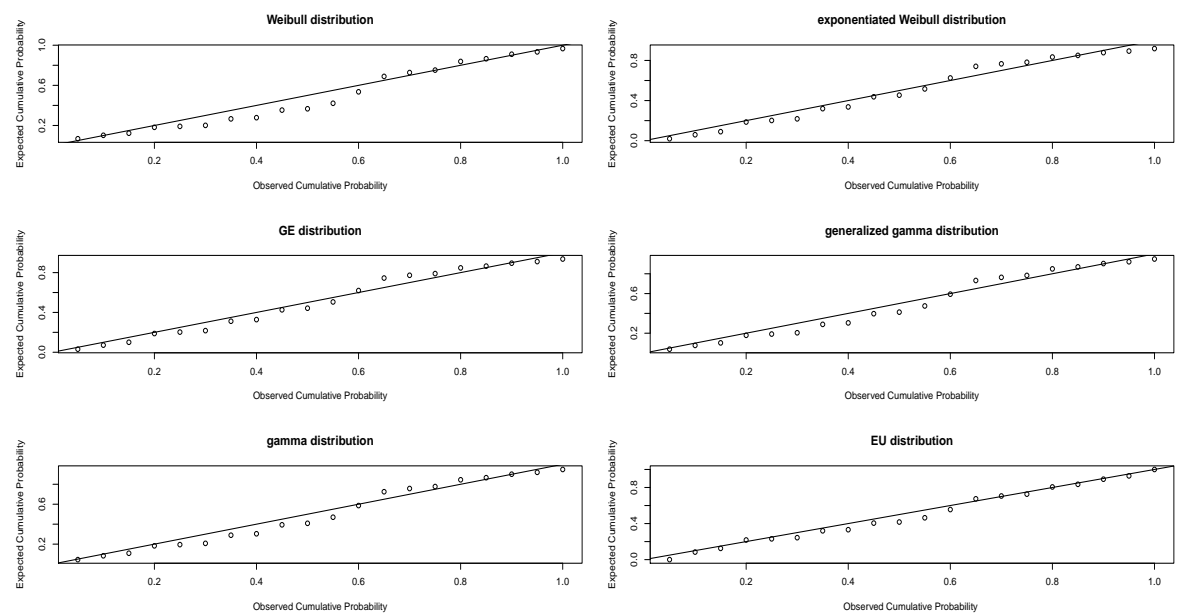

Figure 5. Probability plots for the fit of the Weibull, gamma, GE, E-Weibull, G-gamma and EU distributions to the data. 
Application 3. The following data set are the mean monthly precipitations in one of the cities of Iran (Mashhad) in 2010 (www.climate-charts.com): $33.1,36.4,52,48.8,25.5,3,0.9,0.7,1.5,11.2,15.7,26.9$.

We would like to know what particular distribution can fit to the data. The histogram and the empirical hazard function are shown in Figure 6 . Histogram is monotonically decreasing and the empirical hazard function is increasing. On the basis of our second motivation that was explained in the introduction, the Weibull, gamma and GE distributions are not suitable and we fit EU distribution to the data. The fitted pdf with the empirical histogram and the corresponding probability plot are shown in Figure 7. Both figures suggest that the fit of EU distribution is reasonable. As a further check, we performed a Kolmogorov-Smirnov test for the fit. This test yielded a P-value of 0.3404 .

\section{Conclusion}

We studied EU distribution and mentioned some of its properties. We discussed estimation of the parameters via the maximum likelihood method. Various characterizations of this distribution were presented. Three applications of EU distribution were given to show that this distribution can be used effectively in analysing lifetime data.
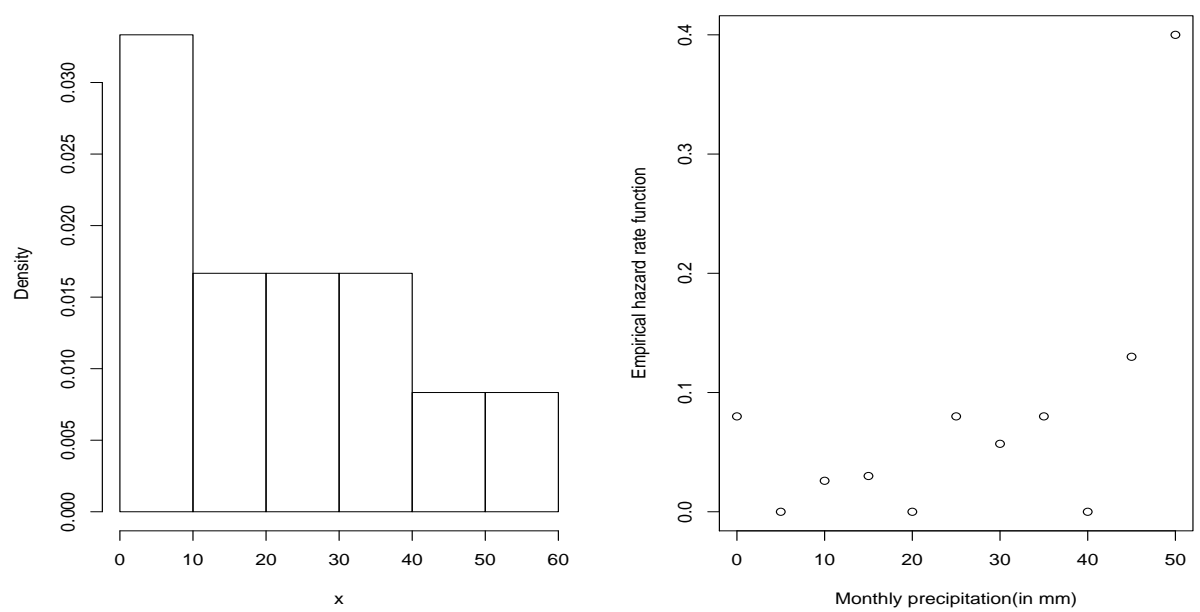

Figure 6. Plots of histogram and Empirical hazard function for monthly precipitations in a city of Iran (Mashhad) in 2010. 

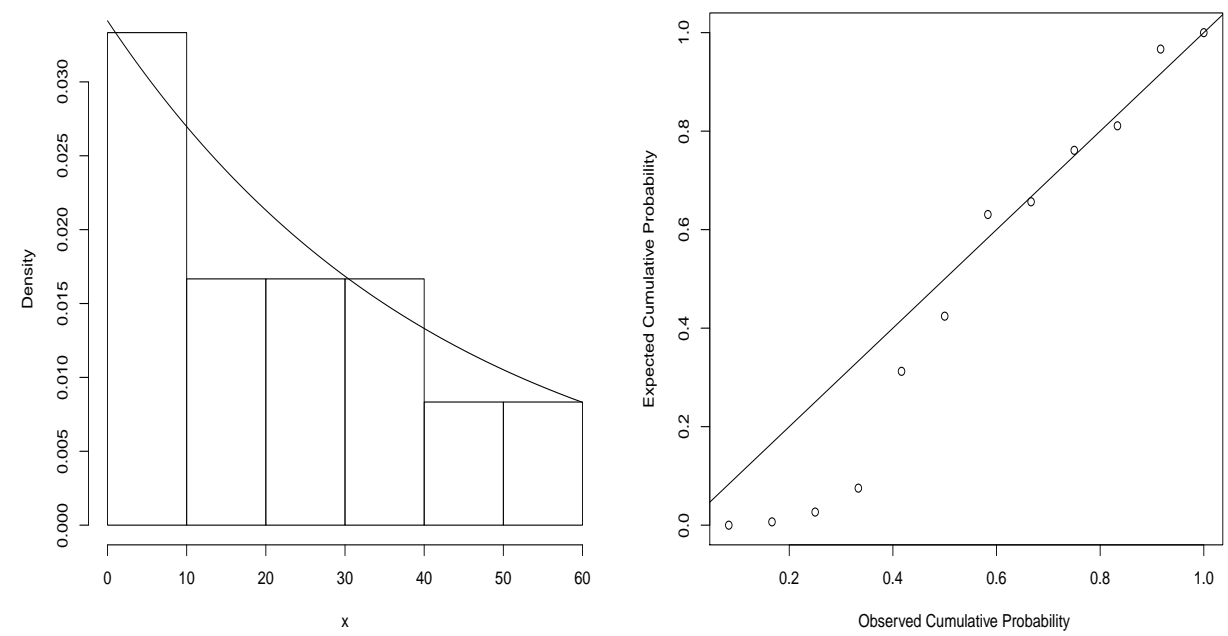

Figure 7. The fitted pdf with the empirical histogram and the corresponding probability plot for monthly precipitations in a city of Iran (Mashhad) in 2010.

\section{Acknowledgement}

The authors would like to thank the referees and the associate editor for constructive suggestions.

\section{References}

Barreto-Souza, W. and Simas, A.B. (2013). The Exp-G Family of Probability Distributions, Brazilian Journal of Probability and Statistics, in press.

Crowder, M.J. (2000). Tests for a Family of Survival Models Based on Extremes, In Recent Advances in Reliability Theory, N. Limnios and M. N, Eds., 307-321. Birkhauser, Boston.

Glänzel, W. (1987). A Characterization Theorem Based on Truncated Momentes and Its Application to Some Distribution Families, Mathematical Statistics and Probability Theory, Vol. B, Reidel, Dordrecht, 75-84.

Glänzel, W. and Hamedani, G.G. (2001). Characterization of Univariate Continuous Distributions, Studia Sci. Math. Hunger., 37, 83-118. 
Hamedani, G.G. (1993). Characterization of Cauchy, Normal and Uniform Distributions, Studia Sci. Math. Hunger., 28, 243-247.

Hamedani, G.G. (2002). Characterization of Univariate Continuous Distributions. II , Studia Sci. Math. Hunger., 39, 407-424.

Hamedani, G.G. (2006). Characterization of Univariate Continuous Distributions. III , Studia Sci. Math. Hunger., 43, 361-385.

Hamedani, G.G. (2010). Characterization of Continuous Univariate Distributions Based on the Truncated Moments of Functions of Order Statistics, Studia Sci. Math. Hunger., 47, 462-484.

Hurvich, C.F. and Tsai, C.L. (1989). Regression and Time Series Model Selection in Small Samples, Biometrika, 76, 297-307.

Lawless, J.F. (2003). Statistical Models and Methods for Lifetime Data, 2nd edition. John Wiley and Sons, Hoboken.

Lehmann, E.L. and Casella, G. (1998). Theory of point estimation, 2nd edition.

Nadarajah, S. and Kotz, S. (2006). The Beta Exponential Distribution, Reliab. Eng. Syst. Saf., 91, 689-697.

Nadarajah, S. and Haghighi, F. (2011). An Extension of the Exponential Distribution, Statistics, 45, 543-558.

Phani, Y., Girija, S.V.S. and Dattatreya Rao, A.V. (2013). Arc Tan-Exponential Type Distribution Induced by Stereographic Projection Bilinear Transformation on Modified Wrapped Exponential Distribution, J. of Appl. Statist. and Informatics (JAMSI), 9, 69-74.

Proschan, F. (1963). Theoretical Explanation of Observed Decreasing Failure Rate, Thechnometrics, 5, 375-383.

Sugiura, N. (1978). Further Analysis of the Data by Akaike's Information Criterion and the Finite Corrections. Comm. Statist., 7, 13-26.

\section{Z. Javanshiri}

Department of Statistics, School of Mathematical Sciences, Ferdowsi University of Mashhad, Mashhad, Iran. email: zo_ja15@um.ac.ir

\section{A. Habibi Rad}

Department of Statistics, School of Mathematical Sciences, Ferdowsi University of Mashhad, Mashhad, Iran. email: ahabibi@um.ac.ir 


\section{G. G. Hamedani}

Department of Mathematics,

Statistics and Computer Science,

Marquette University,

Milwaukee, USA.

email: g.hamedani@mu.edu 